\title{
Computational determination of the largest lattice polytope diameter
}

\author{
Nathan Chadder ${ }^{1}$ \\ Department of Computing and Software \\ McMaster University \\ Hamilton, Canada \\ Antoine Deza $^{2}$ \\ Department of Computing and Software \\ McMaster University \\ Hamilton, Canada
}

\begin{abstract}
A lattice $(d, k)$-polytope is the convex hull of a set of points in dimension $d$ whose coordinates are integers between 0 and $k$. Let $\delta(d, k)$ be the largest diameter over all lattice $(d, k)$-polytopes. We develop a computational framework to determine $\delta(d, k)$ for small instances. We show that $\delta(3,4)=7$ and $\delta(3,5)=9$; that is, we verify for $(d, k)=(3,4)$ and $(3,5)$ the conjecture whereby $\delta(d, k)$ is at most $\lfloor(k+1) d / 2\rfloor$ and is achieved, up to translation, by a Minkowski sum of lattice vectors.
\end{abstract}

Keywords: Lattice polytopes, edge-graph diameter, enumeration algorithm

1 Email: chaddens@mcmaster.ca

2 Email: deza@mcmaster.ca 


\section{Introduction}

Finding a good bound on the maximal edge-diameter of a polytope in terms of its dimension and the number of its facets is not only a natural question of discrete geometry, but also historically closely connected with the theory of the simplex method, as the diameter is a lower bound for the number of pivots required in the worst case. Considering bounded polytopes whose vertices are rational-valued, we investigate a similar question where the number of facets is replaced by the grid embedding size.

The convex hull of integer-valued points is called a lattice polytope and, if all the vertices are drawn from $\{0,1, \ldots, k\}^{d}$, it is referred to as a lattice $(d, k)$-polytope. Let $\delta(d, k)$ be the largest edge-diameter over all lattice $(d, k)$ polytopes. Naddef [7] showed in 1989 that $\delta(d, 1)=d$, Kleinschmidt and Onn [6] generalized this result in 1992 showing that $\delta(d, k) \leq k d$. In 2016, Del Pia and Michini [3] strengthened the upper bound to $\delta(d, k) \leq k d-\lceil d / 2\rceil$ for $k \geq 2$, and showed that $\delta(d, 2)=\lfloor 3 d / 2\rfloor$. Pursuing Del Pia and Michini's approach, Deza and Pournin [5] showed that $\delta(d, k) \leq k d-\lceil 2 d / 3\rceil-(k-3)$ for $k \geq 3$, and that $\delta(4,3)=8$. The determination of $\delta(2, k)$ was investigated independently in the early nineties by Thiele [8], Balog and Bárány [2], and Acketa and Žunić [1]. Deza, Manoussakis, and Onn [4] showed that $\delta(d, k) \geq$ $\lfloor(k+1) d / 2\rfloor$ for all $k \leq 2 d-1$ and proposed Conjecture 1.1.

Conjecture $1.1 \delta(d, k) \leq\lfloor(k+1) d / 2\rfloor$, and $\delta(d, k)$ is achieved, up to translation, by a Minkowski sum of lattice vectors.

In Section 2, we propose a computational framework which drastically reduces the search space for lattice $(d, k)$-polytopes achieving a large diameter. Applying this framework to $(d, k)=(3,4)$ and $(3,5)$, we determine in Section 3 that $\delta(3,4)=7$ and $\delta(3,5)=9$.

Theorem 1.2 Conjecture 1.1 holds for $(d, k)=(3,4)$ and $(3,5)$; that is, $\delta(3,4)=7$ and $\delta(3,5)=9$, and both diameters are achieved, up to translation, by a Minkowski sum of lattice vectors

Note that Conjecture 1.1 holds for all known values of $\delta(d, k)$ given in Table 1 , and hypothesizes, in particular, that $\delta(d, 3)=2 d$. The new entries corresponding to $(d, k)=(3,4)$ and $(3,5)$ are entered in bold. 


\begin{tabular}{|c|cccccccccc|}
\hline$k$ & 1 & 2 & 3 & 4 & 5 & 6 & 7 & 8 & 9 & 10 \\
\hline \hline 1 & 1 & 1 & 1 & 1 & 1 & 1 & 1 & 1 & 1 & 1 \\
2 & 2 & 3 & 4 & 4 & 5 & 6 & 6 & 7 & 8 & 8 \\
3 & 3 & 4 & 6 & 7 & 9 & & & & & \\
4 & 4 & 6 & 8 & & & & & & \\
$\vdots$ & $\vdots$ & $\vdots$ & & & & & & & \\
$d$ & $d$ & $\left\lfloor\frac{3 d}{2}\right\rfloor$ & & & & & & & \\
\hline
\end{tabular}

Table 1

The largest possible diameter $\delta(d, k)$ of a lattice $(d, k)$-polytope

\section{Theoretical and Computational Framework}

Since $\delta(2, k)$ and $\delta(d, 2)$ are known, we consider in the remainder of the paper that $d \geq 3$ and $k \geq 3$. While the number of lattice $(d, k)$-lattice polytopes is finite, a brute force search is typically intractable, even for small instances. Theorem 2.1, which recalls conditions established in [5], allows to drastically reduce the search space.

Theorem 2.1 For $d \geq 3$, let $d(u, v)$ denote the distance between two vertices $u$ and $v$ in the edge-graph of a lattice $(d, k)$-polytope $P$ such that $d(u, v)=$ $\delta(d, k)$. For $i=1, \ldots, d$, let $F_{i}^{0}$, respectively $F_{i}^{k}$, denote the intersection of $P$ with the facet of the cube $[0, k]^{d}$ corresponding to $x_{i}=0$, respectively $x_{i}=k$. Then, $d(u, v) \leq \delta(d-1, k)+k$, and the following conditions are necessary for the inequality to hold with equality:

(1) $u+v=(k, k, \ldots, k)$,

(2) any edge of $P$ with $u$ or $v$ as vertex is $\{-1,0,1\}$-valued,

(3) for $i=1, \ldots, d, F_{i}^{0}$, respectively $F_{i}^{k}$, is a $(d-1)$-dimensional face of $P$ with diameter $\delta\left(F_{i}^{0}\right)=\delta(d-1, k)$, respectively $\delta\left(F_{i}^{k}\right)=\delta(d-1, k)$.

Thus, to show that $\delta(d, k)<\delta(d-1, k)+k$, it is enough to show that there is no lattice $(d, k)$-polytope admitting a pair of vertices $(u, v)$ such that $d(u, v)=$ $\delta(d, k)$ and the conditions (1), (2), and (3) are satisfied. The computational framework to determine, given $(d, k)$, whether $\delta(d, k)=\delta(d-1, k)+k$ is outlined below and illustrated for $(d, k)=(3,4)$ or $(3,5)$. 


\section{Algorithm to determine whether $\delta(d, k)<\delta(d-1, k)+k$}

Step 1: Initialization

Determine the set $\mathcal{F}$ of all the lattice $(d-1, k)$-polytopes $P$ such that $\delta(P)=$ $\delta(d-1, k)$. For example, for $(d, k)=(3,4)$, the determination of all the 335 lattice $(2,4)$-polygons $P$ such that $\delta(P)=4$ is straightforward.

Step 2: Symmetries

Consider, up to the symmetries of the cube $[0, k]^{d}$, the possible entries for a pair of vertices $(u, v)$ such that $u+v=\{k, k, \ldots, k\}$. For example, for $(d, k)=$ $(3,4)$, the following 6 vertices cover all possibilities for u up to symmetry: $(0,0,0),(0,0,1),(0,0,2),(0,1,1),(0,1,2)$, and $(0,2,2)$, where $v=(4,4,4)-u$.

\section{Step 3: Shelling}

For each of the possible pairs $(u, v)$ determined during Step 2, consider all possible ways for $2 d$ elements of the set $\mathcal{F}$ determined during Step 1 to form the $2 d$ facets of $P$ lying on a facet of the cube $[0, k]^{d}$. For example, for $(d, k)=(3,4)$ and $u=(0,0,0)$, we must find 6 elements of $\mathcal{F}, 3$ with $(0,0)$ as a vertex, and 3 with $(4,4)$ as a vertex. In addition, if an edge of an element of $\mathcal{F}$ with $u$ or $v$ as vertex is not $\{-1,0,1\}$-valued, this element is disregarded.

Note that since the choice of an element of $\mathcal{F}$ defines the vertices of $P$ belonging to a facet of the cube $[0, k]^{d}$, the choice for the next element of $\mathcal{F}$ to form a shelling is significantly restricted. In addition, if the set of vertices and edges belonging to the current elements of $\mathcal{F}$ considered for a shelling includes a path from $u$ to $v$ of length at most $\delta(d-1, k)+k-1$, a shortcut between $u$ and $v$ exists and the last added elements of $\mathcal{F}$ can be disregarded.

Step 4. InNER POINTS

For each choice of $2 d$ elements of $\mathcal{F}$ forming a shelling obtained during Step 3, consider the $\{1,2, \ldots, k-1\}$-valued points not in the convex hull of the vertices of the $2 d$ elements of $\mathcal{F}$ forming a shelling. Each such $\{1,2, \ldots, k-1\}$-valued point is considered as a potential vertex of $P$ in a binary tree. If the current set of edges includes a path from $u$ to $v$ of length at most $\delta(d-1, k)+k-1, a$ shortcut between $u$ and $v$ exists and the corresponding node of the binary tree can be disregarded, and the the binary tree is pruned at this node.

A convex hull and diameter computation are performed for each node of the obtained binary tree. If there is a node yielding a diameter of $\delta(d-1, k)+k$ 
we can conclude that $\delta(d, k)=\delta(d-1, k)+k$. Otherwise, we can conclude that $\delta(d, k)<\delta(d-1, k)+k$. For example, for $(d, k)=(3,5)$, no choice of 6 elements of $\mathcal{F}$ forming a shelling such that $d(u, v) \geq 10$ exist, and thus Step 4 is not executed.

\section{Computational Results}

For $(d, k)=(3,4)$, a shelling exists for which path lengths are not decidable by the algorithm without convex hull computations. However, this shelling only achieves a diameter of 7 . For $(d, k)=(3,5)$ the algorithm stops at Step 3 , as there is no combination of 6 elements of $\mathcal{F}$ which form a shelling such that $d(u, v) \geq \delta(2,5)+5$. Thus, no convex hull computations are required for $(d, k)=(3,5)$. A shortcut from $u$ to $v$ is typically found early on in the shelling, which leads to the algorithm terminating quickly. Run on a 2009 Intel ${ }^{\circledR}$ Core $^{\mathrm{TM}_{2}}$ Duo $2.20 \mathrm{GHz}$ CPU, the algorithm is able to terminate for $(d, k)=$ $(3,4)$ and $(3,5)$ in under a minute. Consequently, $\delta(3,4)<8$ and $\delta(3,5)<10$. Since the Minkowski sum of $(1,0,0),(0,1,0),(0,0,1),(0,1,1),(1,0,1),(1,1,0)$, and $(1,1,1)$ forms a lattice $(3,4)$-polytope with diameter 7 , we conclude that $\delta(3,4)=7$. Similarly, since the Minkowski sum of $(1,0,0),(0,1,0),(0,0,1)$, $(0,1,1),(1,0,1),(1,1,0),(0,1,-1),(1,0,-1)$, and $(1,-1,0)$ forms, up to translation, a lattice $(3,5)$-polytope with diameter 9 , we conclude that $\delta(3,5)=9$. Computations for additional values of $\delta(d, k)$ are currently underway. In particular, the same algorithm may determine whether $\delta(d, k)=\delta(d-1, k)+k$ or $\delta(d-1, k)+k-1$ for $(d, k)=(5,3)$ and $(4,4)$ provided the set of all lattice $(d-1, k)$-polytopes achieving $\delta(d-1, k)$ is determined for $(d, k)=(5,3)$ and $(4,4)$. Similarly, the algorithm could be adapted to determine whether $\delta(d, k)<\delta(d-1, k)+k-1$ provided the set of all lattice $(d-1, k)$-polytopes achieving $\delta(d-1, k)$ or $\delta(d-1, k)-1$ is determined. For example, the adapted algorithm may determine whether $\delta(3,6)=10$.

\section{Acknowledgement}

This work was partially supported by the Natural Sciences and Engineering Research Council of Canada Discovery Grant program (RGPIN-2015-06163). 


\section{References}

[1] Dragan Acketa and Joviša Z̆unić, On the maximal number of edges of convex digital polygons included into an $m \times m$-grid, Journal of Combinatorial Theory A 69 (1995), 358-368.

[2] Antal Balog and Imre Bárány, On the convex hull of the integer points in a disc, Proceedings of the Seventh Annual Symposium on Computational Geometry (1991), 162-165.

[3] Alberto Del Pia and Carla Michini, On the diameter of lattice polytopes, Discrete and Computational Geometry 55 (2016), 681-687.

[4] Antoine Deza, George Manoussakis, and Shmuel Onn, Primitive zonotopes, Discrete and Computational Geometry (to appear).

[5] Antoine Deza and Lionel Pournin, Improved bounds on the diameter of lattice polytopes, arXiv:1610.00341 (2016).

[6] Peter Kleinschmidt and Shmuel Onn, On the diameter of convex polytopes, Discrete Mathematics 102 (1992), 75-77.

[7] Dennis Naddef, The Hirsch conjecture is true for $(0,1)$-polytopes, Mathematical Programming 45 (1989), 109-110.

[8] Torsten Thiele, Extremalprobleme für Punktmengen, Master thesis, Freie Universität, Berlin, 1991. 